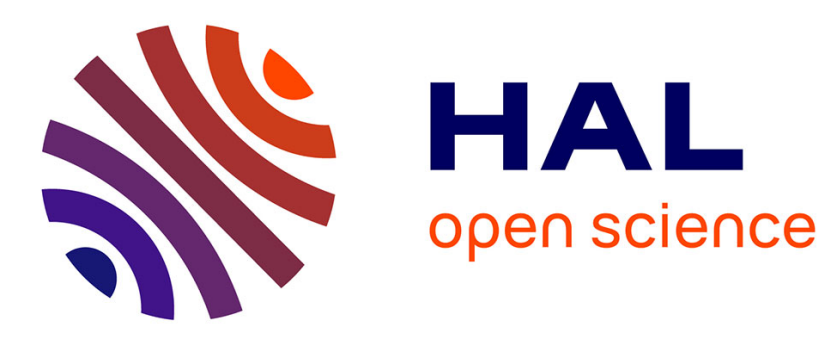

\title{
Des monstres ordinaires
}

Lilian Mathieu

\section{To cite this version:}

Lilian Mathieu. Des monstres ordinaires. Champ Pénal, 2015, XII (Vol. XII), 10.4000/champpenal.9093 . halshs-01326668

\section{HAL Id: halshs-01326668 \\ https://shs.hal.science/halshs-01326668}

Submitted on 8 Jun 2018

HAL is a multi-disciplinary open access archive for the deposit and dissemination of scientific research documents, whether they are published or not. The documents may come from teaching and research institutions in France or abroad, or from public or private research centers.
L'archive ouverte pluridisciplinaire HAL, est destinée au dépôt et à la diffusion de documents scientifiques de niveau recherche, publiés ou non, émanant des établissements d'enseignement et de recherche français ou étrangers, des laboratoires publics ou privés. 
Vol. XII (2015)

Abolitionnisme - Abolitionism

Lilian Mathieu

Des monstres ordinaires

La construction du problème public des clients de la prostitution

\begin{abstract}
Avertissement
Le contenu de ce site relève de la législation française sur la propriété intellectuelle et est la propriété exclusive de l'éditeur.

Les œuvres figurant sur ce site peuvent être consultées et reproduites sur un support papier ou numérique sous réserve qu'elles soient strictement réservées à un usage soit personnel, soit scientifique ou pédagogique excluant toute exploitation commerciale. La reproduction devra obligatoirement mentionner l'éditeur, le nom de la revue, l'auteur et la référence du document.

Toute autre reproduction est interdite sauf accord préalable de l'éditeur, en dehors des cas prévus par la législation en vigueur en France.
\end{abstract}

\title{
revues.org
}

Revues.org est un portail de revues en sciences humaines et sociales développé par le Cléo, Centre pour l'édition électronique ouverte (CNRS, EHESS, UP, UAPV).

Référence électronique

Lilian Mathieu, « Des monstres ordinaires », Champ pénal/Penal field [En ligne], Vol. XII | 2015, mis en ligne le 10

août 2015, consulté le 18 août 2015. URL : http://champpenal.revues.org/9093; DOI : 10.4000/champpenal.9093

Éditeur : Association Champ pénal / Penal field

http://champpenal.revues.org

http://www.revues.org

Document accessible en ligne sur:

http://champpenal.revues.org/9093

Document généré automatiquement le 18 août 2015.

(c) Champ pénal 


\section{Lilian Mathieu}

\section{Des monstres ordinaires}

\section{La construction du problème public des clients de la prostitution}

Comme les "accidents de la route", tenus jadis pour une fatalité, sont devenus "la violence routière", la prostitution est en train de se muer en "violence prostitutionnelle". Comme le mauvais conducteur a désormais à répondre de son comportement, le client prostitueur, qui nourrit un immense marché aux femmes, est aujourd'hui placé en face de ses responsabilités

(Legardinier, 2011).

\section{Introduction}

Émanant d'une militante de l'abolition de la prostitution, la citation placée en exergue à cet article ne peut manquer de faire sourire l'analyste des politiques de la prostitution : le rapprochement entre violence routière et consommation prostitutionnelle évoque immanquablement le travail de Joseph Gusfield sur la construction du problème de l'alcool au volant. Le sociologue américain montrait, dans cet ouvrage devenu classique, combien l'attribution aux conducteurs ivres de la responsabilité des accidents de la route a procédé par exclusion des manières alternatives de poser le problème - par exemple en interrogeant la qualité des véhicules, l'entretien des routes ou encore le manque de transports publics (Gusfield, 2009, 210). Il y étudiait plus spécifiquement les procédés rhétoriques destinés à susciter la croyance (ibid., 30) selon laquelle la baisse du nombre des accidents automobiles passe prioritairement par la sanction des «mauvais conducteurs ».

L'enjeu du présent article est, en s'inspirant de la démarche de Gusfield, d'étudier la manière dont, en France, le client en est venu à être individualisé comme la cause (ibid., 210) du problème prostitutionnel et comment a été suscitée la croyance qu'en s'attaquant à lui ce sont les racines de la prostitution qui seront éradiquées. Il s'agit plus précisément de reconstituer le processus qui, à l'échelle nationale ${ }^{1}$, a vu le recours aux services de prostituées sortir des registres de l'anodin et du privé pour revêtir une dimension doublement problématique et publique. Suivant ici encore Gusfield, on privilégiera l'expression de problème public plutôt que celle de problème social afin de souligner plusieurs caractéristiques du processus ${ }^{2}$. Celuici passe en premier lieu par l'élaboration d'un argumentaire et sa diffusion auprès d'un public qu'il s'agit de sensibiliser à l'importance de l'enjeu et de convertir à l'interprétation qui en est livrée. Il passe également par une interpellation des autorités publiques qui, dans le cas présent, aura été suffisamment efficace pour voir celles-ci s'approprier le problème et entreprendre de lui apporter une réponse politique. On le sait, le problème - désormais totalement constitué des clients de prostituées est en voie de connaître une forme de consécration par le vote d'une proposition de loi visant à la pénalisation de l'achat de services sexuels ${ }^{3}$.

Cet article s'appuie sur les résultats d'une recherche consacrée au mouvement français pour l'abolition de la prostitution (Mathieu, 2014), lequel a joué un rôle majeur - quoique, on le verra, ambivalent et non exclusif - dans la mobilisation pour la pénalisation des clients de prostituées. Cette recherche s'est principalement appuyée sur l'analyse d'une ample documentation écrite, sous forme d'archives associatives, de rapports officiels, d'ouvrages, de presse militante ou grand public, ainsi que d'autres textes. Ont plus particulièrement été étudiées les publications papier et en ligne du Mouvement du Nid (spécialement son trimestriel Prostitution et société), de la Fondation Scelles, d'Attac, du Collectif national pour le droit des femmes, d'Osez le féminisme et du site féministe québécois Sisyphe. Des entretiens ont également été conduits avec des animateurs du Mouvement du Nid et de la Fondation Scelles. L'étude des rapports parlementaires ou institutionnels produits sur la prostitution depuis 2001 a été complétée par une forme de participation observante puisque l'auteur du présent article a été auditionné comme expert à plusieurs reprises (par les députés en 2010, les sénateurs en 2013 et la Commission nationale consultative sur les droits de l'homme en 2014) dans le cours de leur élaboration. 


\section{I - Une problématisation tardive}

On sait depuis Outsiders qu'il revient aux entrepreneurs de morale de faire émerger les problèmes publics en exigeant que soit mis un terme à une situation qui les choque profondément (Becker, 1985). Les croisés de l'abolition de la prostitution ${ }^{4}$ n'ont pas manqué à cette tâche et comptent actuellement parmi les plus fervents promoteurs de la pénalisation des hommes qui fréquentent les prostituées. De fait, le projet d'une disparition de l'offre prostitutionnelle par la répression de sa demande constitue aujourd'hui la principale revendication abolitionniste. Pourtant, il n'en a pas toujours été ainsi et reconstituer les modalités de la conversion de l'abolitionnisme français à la question de la clientèle prostitutionnelle présente plusieurs intérêts. Celui d'une historicisation de la problématisation, tout d'abord : loin de s'imposer sur le mode de l'évidence ou sous l'effet d'un mécanisme logique, c'est par un travail complexe et évolutif de diagnostic, d'interprétation et d'indignation que la figure du client a, tardivement, été placée au centre du « drame prostitutionnel ». Celui, ensuite, d'une complexification d'un processus rien moins que linéaire ou paisible : ce dernier résulte d'une lutte de définition qui a vu s'affronter différents groupes ou fractions aux conceptions divergentes de ce que sont les clients et du traitement auquel il convient de les soumettre. L'enjeu n'est pas mince puisque, comme y insistent Claude Gilbert et Emmanuel Henry, définir un problème (plus exactement, opter pour une définition), c'est se l'approprier (Gilbert, Henry, 2012, 43) - c'est-à-dire, spécialement, s'imposer comme spécialiste incontournable et comme interlocuteur privilégié des pouvoirs publics. Le récit de l'émergence du problème des clients de prostituées est par conséquent celui des recompositions de l'espace de la lutte pour la disparition de la prostitution.

\section{1) Le client, un fantoche}

L'abolitionnisme historique - entendu comme le mouvement né au sein des sectes protestantes anglaises dans la seconde moitié du XIX ${ }^{\mathrm{e}}$ siècle et mobilisé pour l'abolition de toute réglementation de la prostitution - s'est peu intéressé aux clients. Certes, la dénonciation de la prostitution passe par une stigmatisation des privilèges sexuels masculins et la revendication d'une morale unique pour les deux sexes. Leader charismatique de l'abolitionnisme naissant, Josephine Butler réfute par exemple l'idée d'une prostitution exutoire, dans laquelle elle identifie une «doctrine» selon laquelle les femmes sont de simples morceaux de chair destinés à assouvir les désirs masculins les plus vils et (...) les hommes sont, au dernier degré, esclaves de leurs appétits les plus bas (reproduit in Regard, 2013, 248). Mais ce sont avant tout les pouvoirs arbitraires accordés à la police et à la médecine qui constituent la cible principale du mouvement; ainsi que l'indique Alain Corbin, l'objectiffondamental [du projet abolitionniste] n'est point la suppression de la prostitution mais le désenfermement des prostituées, la destruction de tout le système qui tend à créer un milieu marginalisé, hors du droit commun (Corbin, 1982, 332).

Tout entier engagé dans la lutte contre ces institutions-clés du réglementarisme que sont les maisons closes, l'abolitionnisme français de la première moitié du $\mathrm{XX}^{\mathrm{e}}$ siècle n'accorde lui aussi qu'une attention secondaire aux clients. Le raisonnement économique selon lequel c'est l'offre qui crée la demande paraît en effet l'inspirer, et les écrits du mouvement n'ont de cesse de dénoncer les facilités d'accès à la prostitution que garantit sa réglementation. C'est ainsi que, pour l'Union française pour le relèvement de la moralité publique, « la maison de tolérance est une école publique de la dépravation. Par les facilités qu'elle offre, elle provoque le besoin qu'elle prétend seulement satisfaire » (Union française..., 1936, 3). Cette perspective dessine une conception dénaturalisée de la sexualité masculine, qui n'obéit pas à des pulsions instinctuelles mais est façonnée et orientée par une offre commerciale spécifique. Les effets pernicieux de ces «laboratoires du vice » que sont les maisons de tolérance se font particulièrement sentir parmi les jeunes, précocement incités et habitués à une dépravation dont ils peineront plus tard à se défaire, et qui représente une contre-éducation qui bafoue tous les efforts faits par la famille (Gemähling, 1933, 54). L'enjeu est donc de s'attaquer aux instances qui construisent la demande de prostitution mais pas directement à ceux qui l'incarnent. Cet évitement s'explique en outre par un respect vigilant des libertés individuelles 
qui constitue, depuis ses origines, un des principes fondateurs de l'abolitionnisme. L'Union temporaire contre la prostitution réglementée et la traite des êtres humains refuse ainsi de s'attaquer à la prostitution en tant que telle car cela est impossible « sans s'immiscer dans la vie privée des gens ; sans tomber dans l'arbitraire » (Union française..., 1936, 20).

Issu du catholicisme social et appelé à devenir la principale association abolitionniste française, le Nid ne déroge pas à cette conception une fois obtenue l'interdiction des établissements de prostitution par la loi du 13 avril 1946 : « On veut faire croire aux parents que la réouverture préservera leurs fils des sollicitations du trottoir. Mince argument, et facile à retourner. Combien de jeunes, en effet, qui, par peur (des maladies surtout) n'auraient jamais osé aller avec une fille du trottoir, se laisseront tenter par une "maison" offrant des "garanties" (...). En outre, les hommes qui ont été à la caserne avant 46 le savent (...), la simple présence d'une maison de tolérance est une provocation à la débauche. Loin de soulager l'obsession, elle peut l'entretenir et la développer » (Moissons nouvelles, 1955, $\mathrm{n}^{\circ}$ 14-15, 34-35). Cette thèse d'une plasticité des désirs masculins en quelque sorte déterminés par l'état de l'offre sexuelle, s'accompagne à la même époque de l'adoption d'un cadre d'interprétation de la prostitution à dominante psycho-pathologique. Si, comme on l'a montré ailleurs (Mathieu à paraître), les femmes prostituées sont dépeintes comme des « débiles mentales » marquées par une série de carences affectives et morales, les clients sont également appréhendés comme psychologiquement influençables, voire déficients ${ }^{5}$. C'est leur immaturité qui est pointée dans le trimestriel du Nid, Moissons nouvelles : «Un homme de 40 ans qui a recours aux prostituées manifeste un état d'évolution de sa sexualité correspondant environ à 17 ou 18 ans » (1954, n -27, 56). Les clients seraient ainsi «hypoévolués au point de vue sexuel et affectif et souvent, par surcroit, névrosés ou déséquilibrés » et exigeraient des « traitements psychiatriques et psychanalytiques appropriés » $\left(1964, \mathrm{n}^{\circ} 51,116\right)$.

Cette tonalité à la fois psychologisante et misérabiliste marque durablement la conception abolitionniste du client. En 1973, un psychiatre du Nid, le docteur Safar, le décrit comme un désengagé affectif (Safar, 1973, 25) et en 1996 Claudine Legardinier peut encore le dépeindre comme poussé par le mal-être et la difficulté à communiquer mais se trompant lorsqu'il cherche une réponse à ses problèmes dans une consommation sexuelle qui ne serait qu'un marché de dupes où client et prostitué(e) sont floués, pendant que le proxénète ramasse les dividendes (Legardinier, 1996, 11). Si la même auteure évoque l'opposition entre les options d'une répression des clients et de leur responsabilisation par l'éducation, elle se garde encore de trancher.

\section{2) Une problématisation féministe}

On le voit, l'abolitionnisme n'a pendant longtemps accordé qu'une attention secondaire au client, sorte de fantoche aux désirs manipulés par les proxénètes et uni à la prostituée par un malaise existentiel commun ${ }^{6}$. Ce n'est donc pas de ce mouvement qu'émergera, à compter des années 1990, la première problématisation du rôle du client dans la perpétuation du phénomène prostitutionnel mais de l'espace de la cause des femmes (Bereni, 2012), à la même époque en phase de remobilisation et de reconstitution organisationnelle ${ }^{7}$.

Le mouvement des femmes français n'avait jusqu'alors manifesté que peu d'intérêt pour la prostitution, hormis en 1975 un soutien conjoncturel aux prostituées en lutte contre la répression policière (Mathieu, 2001). Si les principales intellectuelles féministes françaises - Simone de Beauvoir et Colette Guillaumin, notamment - ont consacré quelques pages au sujet, c'est surtout en faisant de la situation de la prostituée le paroxysme de la condition de l'ensemble des femmes, sans lui consacrer de réflexion spécifique. Les thématiques sexuelles ont longtemps été monopolisées par les analyses à dominante psychanalytique du courant « différentialiste » et les principaux combats du féminisme - pour le contrôle de la fertilité et contre le viol, spécialement - l'ont en outre amené à envisager la sexualité au prisme des risques auxquels elle expose les femmes. Le mouvement des femmes français est également longtemps resté à l'écart des sex wars qui ont vu, dans les années 1980 aux États-Unis, s'opposer opposantes résolues à la pornographie et à la prostitution définies comme des 
violences sexistes et promotrices d'une émancipation des femmes par diversification de leur répertoire sexuel.

Deux entrepreneures de morale vont jouer un rôle déterminant dans l'appropriation du thème prostitutionnel par le féminisme français. La première est la journaliste Florence Montreynaud qui publie en 1993 un livre iconographique consacré à la prostitution, dans lequel elle interroge le regard du client sur cette activité (Montreynaud, 1993). Cet ouvrage, salué par le Mouvement du Nid, est non seulement à l'origine d'une attention plus vive de son auteure pour les clients mais également de son rapprochement avec l'abolitionnisme. Montreynaud tient à la fin des années 1990 une rubrique régulière dans le trimestriel du Nid Prostitution et société (désormais $P \& S$ ), intitulée « Payer pour ça », dans laquelle elle se livre à une dénonciation sarcastique de cette pratique spécifiquement masculine qu'est l'achat de « services sexuels ». Son accès aux médias et la tonalité humoristique de ses prises de position lui permettent d'exprimer largement une dénonciation de toute négociation monétaire de l'intimité du corps humain (Montreynaud, 1999).

La seconde entrepreneure de morale est la sociologue Marie-Victoire Louis. Antérieurement surtout spécialisée en sociologie du travail, elle commence à s'intéresser à la prostitution au début des années 1990, tout d'abord comme observatrice des débats sur le sujet interne aux arènes institutionnelles européennes. Celles-ci sont en effet bousculées par l'opposition entre les approches de la prostitution respectivement envisagées puis adoptées par la Suède et la Hollande : la première considère la prostitution comme une violence sexiste dont les auteurs - les clients - doivent être sanctionnés tandis que la seconde entreprend une normalisation du « marché du sexe » par sa reconnaissance comme métier à part entière. Louis ne se contente pas de dénoncer l'approche hollandaise comme une remise en cause du principe, pourtant universel, selon lequel le corps humain est inaliénable (Louis, 1999). Elle se pose, au sein du féminisme et au-delà, en lanceuse d'alerte devant ce qu'elle perçoit comme une entreprise, menée par la Hollande, de conversion d'autres pays à la reconnaissance du « travail du sexe ». D'où, notamment, ses attaques contre les associations de santé communautaire qui, à partir d'une action de prévention du VIH à destination des prostituées, revendiquent la reconnaissance d'un tel « travail».

Les prises de positions de Montreynaud et de Louis connaissent une large diffusion au sein de l'espace de la cause des femmes, et spécialement du CNDF qui, par exemple, s'approprie l'expression de système prostitutionnel forgée par Louis (2004) ${ }^{8}$. Montreynaud est quant à elle invitée en 2002 au Forum du CNDF où elle défend l'idée selon laquelle s'il n'y avait pas de client, il n'y aurait pas de prostitution (Montreynaud, 2002, 195). Interviennent au même atelier deux autres entrepreneures de morale, actrices majeures du rapprochement entre abolitionnisme et féminisme. La première est Malka Marcovich, représentante du Mouvement pour l'abolition de la prostitution et de la pornographie (MAPP) qui est l'antenne française de la Coalition against trafficking in women (CATW) fondée par l'universitaire américaine Kathleen Barry. Marcovich est en 2002 l'auteure d'un rapport pour la Commission nationale contre les violences envers les femmes intitulé Le système de la prostitution : une violence à l'encontre des femmes, lui aussi largement relayé au sein des groupes féministes et dans lequel elle décrit l'acte sexuel marchand comme la clé de voûte de la persistance des inégalités entre hommes et femmes (Marcovich, 2002, s.p.). La seconde entrepreneure présente au Forum du CNDF est la journaliste du Nid Claudine Legardinier, dont les positions - comme celles de son association - connaissent à la même époque une sensible évolution. L'article sur la prostitution qu'elle rédige pour le Dictionnaire critique du féminisme s'achève en effet sur une présentation cette fois favorable de la politique suédoise de pénalisation des clients, sur laquelle il n'est que temps de s'arrêter (Legardinier, 2000).

\section{3) Le modèle suédois}

La problématisation de la contribution des clients au phénomène prostitutionnel n'aurait pas, en France, pris le tour qu'elle a connu si la politique adoptée en 1999 par la Suède n'avait pas bénéficié au même moment d'une ample promotion. La Suède a en effet été le premier pays à faire de l'achat de services sexuels un délit passible d'une peine de 50 jours d'amende 
(leur montant est en Suède calculé en fonction des revenus) et de six mois de prison. Cette politique pénale a pris place parmi un ensemble de dispositions visant à lutter contre les violences sexistes adoptées par le gouvernement social-démocrate de l'époque, et ce sont des parlementaires féministes qui en ont été les principales promotrices. Elle s'appuie sur l'idée que "l'égalité des sexes restera hors d'atteinte tant que les hommes achèteront, vendront, exploiteront des femmes et des enfants en les prostituant ${ }^{9}$. Plus globalement, elle envisage la prostitution comme une violence sexiste dont les principaux auteurs, les clients, doivent par conséquent être sanctionnés.

Les féministes françaises sont les premières à s'intéresser à la politique suédoise. La nouvelle définition de la prostitution comme violence sexiste rencontre un écho immédiat au sein d'un mouvement qui, on l'a dit, s'est historiquement constitué dans le combat contre les violences faites aux femmes. Mais la légitimation du «modèle suédois » tient surtout à un actif travail de promotion mené dès le début des années 2000 par le gouvernement suédois lui-même dont les représentantes féministes - ou fémocrates, pour reprendre l'expression popularisée par Lee Ann Banaszak (2010) - ont investi une multiplicité d'instances de débat et de réflexion où elles ont présenté les acquis de la pénalisation des clients. Pour ce qui est de la France, elles étaient par exemple présentes au forum du CNDF de 2002 et, l'année suivante, à l'Assemblée européenne des femmes, parallèle au Forum social européen ainsi qu'au colloque «Politiques urbaines face à la prostitution » (animé par C. Legardinier et M. Marcovich), tout en assurant un séminaire sur la question à l'ambassade de Suède. Le caractère systématique de cette promotion, par ailleurs appuyée et relayée par le Lobby européen des femmes, se repère à la récurrence des mêmes «éléments de langage » dans toute présentation de l'innovation suédoise : division par deux du nombre de prostituées de rue, soutien accru de la population à une mesure initialement perçue avec réticence, écoutes téléphoniques de mafieux révélant leur désintérêt pour un pays où la prostitution ne serait plus rentable, jusqu'au nom de l'ensemble législatif au sein duquel s'intégrait la pénalisation des clients - « la paix des femmes »assimilant la prostitution à une pratique de guerre. Montreynaud, Marcovich, Louis et Legardinier se feront toutes les ardentes promotrices du modèle suédois au sein de l'espace français de la cause des femmes, et même au-delà. Les connexions entre certaines fractions du mouvement des femmes et l'extrême gauche favorisent sa promotion à l'intérieur du mouvement altermondialiste, et spécialement au sein d'Attac. La commission «Genre » de cette association publie en 2008 un court ouvrage consacré aux liens entre mondialisation et prostitution qui s'achève en avançant que « la pénalisation des clients/ prostitueurs constitue un élément clé de l'urgente politique à mettre en œuvre (...) pour mettre fin au plus vieux système d'exploitation du monde » (Attac, 2008, 103-104).

$\mathrm{Si}$ les féministes se rallient rapidement à la pénalisation des clients, c'est au sein de l'abolitionnisme que celle-ci rencontre le plus de résistances. L'hostilité à l'égard de toute mesure coercitive a longtemps été de mise au sein du Nid, qui a toujours fait de la pédagogie et de la responsabilisation les maîtres mots d'une démarche misant avant tout sur l'éveil des consciences. «La disparition du client, ce n'est pas monter les enchères de la répression » pouvait-on lire en 1990 dans le $n^{\circ} 91$ de $P \& S$, et la même position était toujours de mise en 2002 : «La pénalisation des clients apparaît à tort comme la panacée qui évite d'aborder la question dans toute sa complexité. Par ailleurs, pénaliser les clients reviendrait à opter pour une mesure prohibitionniste. Le MdN a toujours été opposé aux mesures sur le racolage qui enfoncent les personnes prostituées dans la mort sociale et les condamnent à plus d'exploitation, y compris de la part de l'État. En toute logique, il les refuse également pour les clients $\gg\left(P \& S, 2002, \mathrm{n}^{\circ} 139,27\right)$. Mais en 2007 , le conseil national du Nid se range en faveur de «l'établissement d'une norme légale interdisant l'achat d'un acte sexuel». On perçoit le déchirement entre pédagogie et répression que ressent alors l'association à la lecture de cette justification pour le moins paradoxale d'une « sanction qui ne devra (...) pas être utilisée à des fins punitives mais au contraire comme un levier de prévention et de responsabilisation » ( $P \& S$, 2007, $\mathrm{n}^{\circ} 156,28-29$ ). Deux ans plus tard, cette dimension pédagogique s'est estompée et c'est avec fermeté que le MdN exige l'interdiction de l'achat de tout service sexuel par des clients avant tout désignés comme des agresseurs $\left(P \& S, 2009, \mathrm{n}^{\circ} 166,25\right)$. 
C'est donc à un revirement complet du principal groupe abolitionniste français que l'on a assisté en l'espace de quelques années. Plusieurs raisons peuvent l'expliquer. Une première est le relatif rajeunissement d'une association dont la génération des fondateurs, socialisée au sein du catholicisme social, s'efface au profit de nouveaux militants porteurs d'autres références politiques et, notamment, davantage réceptifs aux positions féministes. Une deuxième est la recomposition que connaît au même moment l'espace abolitionniste avec la création en 1993 d'une nouvelle organisation, la Fondation Scelles, dont l'audience et l'influence s'élargissent à partir de $2000^{10}$. Héritière directe des Équipes d'action contre le proxénétisme, la Fondation Scelles est de sensibilité plus conservatrice que le Nid et, surtout, davantage adepte des options pénales ${ }^{11}$. C'est fort logiquement qu'elle soutient d'emblée la pénalisation des clients, tout en exposant le Nid à apparaître plus timoré. On ne peut donc exclure que ce soit, au moins en partie, sous l'effet d'un radical flank effect - le risque pour les tenants de positions modérées d'être marginalisés par ceux que leur radicalité place à la pointe de la lutte (Haines, 2013) que le Nid se soit rallié à une option qu'il refusait encore peu auparavant. Une troisième raison, que l'on abordera plus loin, est l'appropriation de la pénalisation des clients par le champ politique à partir du début des années 2000 , ce qui a contribué à lui conférer une légitimité et une plausibilité nouvelles.

On le voit, la problématisation de la question des clients de prostituées est non seulement récente au sein de l'abolitionnisme français mais s'est surtout réalisée à son corps défendant, sous l'effet d'une mobilisation, inédite au moins dans son ampleur, du féminisme français sur cette question. Le revirement du Nid en faveur d'une option qu'il a longtemps rejetée montre que cette problématisation est le résultat d'une lutte de définition qui a vu celle défendue par le féminisme l'emporter sur celle traditionnellement portée par l'abolitionnisme. Plus encore, c'est à condition d'intégrer une large part des schèmes et du vocabulaire féministes qu'une association catholique - et à ce titre perçue avec méfiance - comme le Nid a su se rendre acceptable comme partenaire par le mouvement des femmes.

\section{II - La construction d'une monstruosité}

Un biais intellectualiste largement répandu au sein de la sociologie des mouvements sociaux, et spécialement au sein de la théorie des cadrages héritée d'Erving Goffman, accorde un poids déterminant à la « robustesse » et à la « résonance » des discours militants dans le succès des mobilisations. Dans cette optique, seraient plus « efficaces» les argumentations militantes les plus cohérentes et raffinées. La critique de cette croyance naïve dans une toutepuissance des idées (Mathieu, 2002) ne doit pas pour autant conduire à dédaigner l'analyse des argumentations que diffusent les organisations de mouvement social. En premier lieu parce que leur élaboration relève d'une pratique militante à part entière, ensuite parce que s'y expriment - mais de manière le plus souvent implicite ou inconsciente - certains des ressorts sociaux de l'engagement de ceux et celles qui s'y consacrent. L'élaboration des arguments, le choix du vocabulaire, le cheminement des démonstrations... ne se font pas au hasard mais en puisant dans un stock de références culturelles dont le contenu est socialement déterminé. Il convient donc, en suivant une fois encore l'exemple de Gusfield, de s'arrêter sur les formes rhétoriques qui ont façonné la problématisation de la question des clients.

\section{1) Un monstre...}

21 Les procédés rhétoriques de problématisation comportent deux dimensions (Gusfield, 2009, 9sq.). L'une, cognitive, vise l'établissement de faits irréfutables, qu'il s'agit de porter à la connaissance d'un public. L'autre, morale, porte un jugement normatif sur ces faits en les présentant comme indignes, repoussants et condamnables. Abolitionnistes et féministes, désormais unis, vont s'y employer au travers d'une production discursive qui, pour être relativement abondante, n'en est pas moins homogène : c'est globalement le même portrait des clients - les décrivant comme des monstres ordinaires - qui est dressé au sein des différentes fractions et courants de la mobilisation pour leur pénalisation.

22 Une attention préalable à l'analyse de ces discours doit être portée à leurs supports. On peut tout d'abord remarquer qu'au désintérêt abolitionniste pour les clients succède, à partir 
du début des années 2000, une intense focalisation sur leur contribution au phénomène prostitutionnel. Prostitution et société leur consacre ainsi des dossiers à répétition : "Clients de la prostitution : le grand secret » (2001, $\left.\mathrm{n}^{\circ} 134\right)$; «Faut-il pénaliser les clients de la prostitution ? » (2002, $\left.\mathrm{n}^{\circ} 139\right)$; « On ne naît pas client, on le devient » (2004, $\left.\mathrm{n}^{\circ} 146\right)$; «L'image du client en France » (2004, $\left.\mathrm{n}^{\circ} 147\right)$; « Clients de la prostitution : nouveaux regards, nouvelles politiques » (2006, $\left.\mathrm{n}^{\circ} 152\right)$; «Prostitueurs : état des lieux » (2008, n - 163). Le Nid entreprend à la même époque une enquête sur les clients, pilotée par C. Legardinier en collaboration avec le sociologue Saïd Bouamama, dont il assure la promotion et le commentaire dans son trimestriel (Bouamama, Legardinier, 2006). On remarque ensuite que cette production discursive émane d'un groupe stable et restreint de signataires récurrents. Quoique prétendant apporter des connaissances inédites sur le sujet et donc faire œuvre scientifique, elle reste en outre cantonnée à des supports de diffusion militants. Les ouvrages, revues et sites internet d'organisations de mouvement social ou à nette coloration « engagée » constituent ainsi les principaux canaux de diffusion d'une connaissance dont les sources ou conditions de production sont rarement explicitées et qui ignore le plus souvent les connaissances scientifiques disponibles ${ }^{12}$.

La double dimension cognitive et morale s'exprime tout d'abord dans le constat récurrent que les clients seraient mal connus : publiciser un savoir nouveau reviendrait à démasquer ceux que l'anonymat et le silence protègeraient. Ainsi la quatrième de couverture du livre de Bouamama et Legardinier annonce-t-elle que les auteurs « rompent ainsi le silence qui a toujours pesé sur les pratiques des clients pour interroger leur responsabilité dans la montée de la traite des êtres humains, du tourisme sexuel et des violences contre les femmes, mais aussi dans le maintien de l'inégalité entre les sexes ». De même l'article de Christine dans le dossier d'Alternative libertaire sur le sujet s'ouvre-t-il sur le constat que les clients ont été longtemps "les grands inconnus" du système prostitutionnel (Christine, 2010,11) tandis que $P \& S$ annonce dans son $\mathrm{n}^{\circ} 163(2008$, p20) que le client est resté « sans visage et sans nom pendant des siècles ». Il ne s'agit pas seulement d'apporter un savoir inédit mais d'amener à envisager sous un jour nouveau une réalité que sa connaissance actuelle ne permet pas d'appréhender dans sa « vérité », ce qui implique l'adoption d'un vocabulaire nouveau. Bouamama et Legardinier indiquent dans l'avant-propos de leur livre qu'ils n'useront dans ces pages du mot "client" que faute de mieux, sans nous encombrer des guillemets qu'il requiert. Ce terme impropre constitue en effet un euphémisme et une normalisation insidieuse du proxénétisme et du commerce des corps. La langue française, ce n'est pas un hasard, n'a rien d'autre à nous offrir (Bouamama, Legardinier 2006, 7). Même constat chez Montreynaud qui récuse (...) le mot "client" car insuffisamment stigmatisant, à ses yeux, à la différence du symétrique «pute». Constatant que prostituée est un participe passé qui suppose une action, elle propose le terme de prostituant pour désigner la personne qui prostitue, par qui l'acte de prostitution est commis. La même auteure suggère également le terme de putanier qui vient de la même racine [que pute], d'un mot latin qui signifie "puer" parce que tout ça c'est dégueulasse (Montreynaud, 2002, 196). Mais c'est le terme de prostitueur qui s'est progressivement imposé dans la rhétorique du mouvement afin de désigner en même temps que stigmatiser ceux qui, en payant des prostituées, entretiennent leur activité.

La normativité du regard porté sur les clients s'exprime avant tout par leur réduction à une intra-humanité, voire à une animalité. C'est ainsi une sexualité instinctive et pulsionnelle qui parait guider des clients qu'il conviendrait dès lors de « civiliser ». On lit ainsi en 2009, dans le $n^{\circ} 166$ de $P \& S$, que « la nature violente du rapport prostitutionnel ruine les démarches entreprises pour "civiliser" les prostitueurs ». Le même trimestriel n'hésite pas à titrer dans son $\mathrm{n}^{\circ} 153(2006,2)$ : « Foot, bière et putes... Les barbares sont de retour » et à employer en $2011\left(n^{\circ} 172,19\right)$ le terme de «prédateurs » tandis que Legardinier mobilise celui de bestialité (Legardinier, 2012, 51). Marcovich choisit quant à elle le terme évocateur de hordeChaque week-end, des hordes d'hommes finlandais viennent à Tallin (...) pour acheter alcool et femmes (Marcovich, 2006, 481) - tandis que c'est le verbe «s' abattre » qu'utilise la Marche mondiale des femmes pour stigmatiser « ceux qui, comme des charognes [sic], vont s'abattre 
sur les jeunes filles ou femmes pour les transformer en marchandise facile et bon marché venant satisfaire une sexualité masculine machiste et dominatrice ${ }^{13}$.

Cette monstruosité des clients s'exprimerait par de multiples caractéristiques de leurs attitudes et comportements. Ainsi du racisme, détecté dans la propension des clients à choisir des prostituées en fonction de critères ethniques : Les stéréotypes sexuels d'ordre raciste et colonialiste jouent un rôle majeur : femmes asiatiques présentées comme attentionnées et soumises, les Africaines comme animales et sexuellement puissantes, les Latino-américaines comme libres et faciles (Bouamama, Legardinier, 2006, 71). Ainsi également de «l'ignorance, la méconnaissance d'autrui et l'irresponsabilité » considérées par le Nid comme « au cœur du clientélisme prostitutionnel » $\left(P \& S, 2007, \mathrm{n}^{\circ} 156,28\right)$. Cette ignorance s'expliquerait, principalement, par les carences d'un apprentissage sexuel dont la pornographie serait le principal, voire le seul, support : La solitude face aux questionnements liés à la sexualité pousse vers les seules réponses disponibles, qui sont inégalitaires et d'ordre mécanique, de la pornographie (Legardinier, 2012,50) et, par conséquent, la sexualité des clients révèlerait « un imaginaire modelé par la pornographie $»^{14}$.

Enfin, et plus fondamentalement, le recours à la prostitution relèverait de la violence. Celle-ci se voit conférer une sorte de double statut, à la fois pratique et ontologique. Les clients seraient en effet nombreux à commettre des violences à l'encontre des prostituées qu'ils fréquentent, et Legardinier relève que la majorité des actes violents subis par les personnes prostituées violences verbales, physiques, sexuelles - sont le fait des clients (Legardinier, 2012, 49). Mais ils se rendraient plus fondamentalement coupables de la violence que serait en elle-même la prostitution ${ }^{15}$. Ainsi $P \& S$ avance $\left(2008, \mathrm{n}^{\circ} 163,13\right)$ que « le sexe marchand est une violence incomparable pour celle ou celui qui la subit », tandis que la Ligue communiste révolutionnaire l'envisage comme « la forme de violence la plus extrême de la domination masculine » (LCR, 2006, 5) et Osez le féminisme argue que « la prostitution - indépendamment de ses conditions d'exercice - est en soi une violence car c'est d'abord un rapport sexuel désiré par un seul des protagonistes : le client prostitueur ${ }^{16}$.

Le client connaît, au sein du discours abolitionniste-féministe, une incarnation privilégiée sous les traits du supporter de football. Une sociabilité présentée comme exclusivement masculine couplée à une consommation supposée élevée d'alcool seraient ainsi des facteurs prédisposant à la « consommation » de prostituées. L'association s'est affirmée en 2006 à l'occasion de la pétition «Acheter du sexe n'est pas un sport », lancée à l'occasion du Mondial de football organisé en Allemagne, pour s'indigner que 40000 prostituées étrangères soient alors « importées » pour répondre à la demande des supporters ${ }^{17}$. Le succès de cette pétition et, plus globalement, de l'association entre sports populaires masculins et recours à la prostitution, tient sans doute à ce qu'elle incriminait les amateurs de football (i.e. le sport le plus apprécié au sein des fractions masculines des classes populaires, qu' une condescendance de classe présente volontiers comme plus frustes, spécialement en matière de rapports entre les sexes, que des couches sociales davantage intellectualisées). Et c'est bien un portrait socialement cernable - et socialement généré - que dessine la conception du client que livrent abolitionnistes et féministes : violent, sexiste, raciste, ignorant, à la sociabilité principalement masculine, consommateur d'alcool ${ }^{18}$ et amateur de football, le client a tous les traits du «beauf », c'est-àdire de la représentation que la bourgeoisie cultivée se fait des fractions les plus culturellement et économiquement démunies des classes populaires ${ }^{19}$.

\section{2 ) ... ordinaire}

Loin d'être cohérent, le discours abolitionniste et féministe recèle des contradictions. Que les traits qui dépeignent les clients soient socialement situés n'empêche pas l'invocation parallèle de leur caractère ordinaire et banal, condition d'une généralisation et d'une légitimation de leur dénonciation (Boltanski, 1990). Le client ce serait ainsi « monsieur tout le monde » et aucune variable ne permettrait véritablement de le spécifier. Ainsi, selon Christine d'Alternative libertaire, ce sont des hommes ordinaires de tous âges, toutes professions, classes sociales, nationalités et "races" (Christine, 2010, 11). Même assertion chez Bouamama et Legardinier qui soulignent que les hommes que nous avons rencontrés 
sont des hommes ordinaires. Ils n'entrent pas dans une catégorie spécifique que l'on pourrait délimiter par des caractéristiques objectives (Bouamama, Legardinier, 2006, 111). La Fondation Scelles est au diapason en affirmant - avec cette innovation qu'est la mention des clientes - que mariés ou non, ce sont des hommes ou des femmes qui font partie de toutes les classes sociales, de tous âges et de toutes origines. Il est impossible de dresser un portrait type du client (Charpenel, 2012, 161). La figure de l'homme non-client, pourtant majoritaire dans la population (Bajos et al., 1997), paraît s'évaporer face à un nouvel avatar de la banalité du mal.

L'insistance sur le caractère « ordinaire » mais aussi insaisissable de l'homme client remplit plusieurs objectifs. Un premier est une sensibilisation du public et sa conversion à l'importance de la cause de la pénalisation. Le recours à la prostitution ne serait pas un problème circonscrit à une fraction de population et qu'il serait aisé de juguler, mais constitue un mal d'autant plus dangereux qu'il est diffus, répandu et anonyme. Pour Bouamama et Legardinier, l'idée de l'existence d'une minorité de pervers (ou de malades, d'accidentés de l'existence) est donc erronée (Bouamama, Legardinier, 2006, 111). Un deuxième objectif, qui découle du précédent, est d'imputer aux clients la responsabilité de leurs actes en leur interdisant de les justifier par l'invocation d'une contrainte impérative - ce que Montreynaud $(2002,198)$ résume par un lapidaire on n'a jamais vu une couille éclater. Legardinier réfute à sa suite les autojustifications des clients, invoquant par exemple la solitude ou des besoins sexuels irrépressibles, pour à l'inverse pointer la volonté de domination qui serait seule au principe de l'achat de services prostitutionnels (Legardinier, 2012, 49). On comprend dès lors que les abolitionnistes se soient vigoureusement mobilisés contre toute forme d'assistance sexuelle aux handicapés : la reconnaissance que certains pourraient ne pas avoir d'alternative à une forme marchande et professionnalisée de sexualité ne pouvait que compromettre cette dimension de leur argumentation.

Legardinier insiste sur ce qui lui apparaît comme la principale caractéristique de ces «hommes ordinaires », à savoir leur attachement à tous les poncifs liés aux identités sexuées, et d'une manière générale à un ordre patriarcal traditionnel $(2012,49)$. Les clients seraient ainsi attachés à l'ordre traditionnel et inégalitaire des rapports entre les sexes, qui légitimerait et déculpabiliserait leur pratique. En découlerait leur hostilité au féminisme et, plus globalement, à toute revendication d'émancipation des femmes : les clients «se présentent comme des victimes : de leurs "besoins" et des femmes - trop exigeantes, trop compliquées, trop égoïstes, trop provocantes, trop libres, dotées de trop de pouvoir » (Attac, 2008, 55). Quoique contredisant l'évocation d'une sexualité «bestiale » et «barbare », cette étiologie du recours aux prostituées par une socialisation masculine patriarcale permet de l'ancrer dans un contexte historiquement situé. Par suite, si cette conduite est une pratique profondément culturelle (Bouamama, Legardinier, 2006, 67), elle n'est pas inéluctable mais peut être modifiée par le changement de ses conditions sociales d'engendrement - objectif que vise, précisément, sa pénalisation. Le Nid affirme ainsi que « c'est bien la culture, l'apprentissage des rôles masculin et féminin qui crée et entretient le système prostitutionnel. Un réel changement à long terme ne fera donc pas l'économie de la mise en place d'une véritable force d'initiative politique » $\left(2008, \mathrm{n}^{\circ} 163,31\right)$.

Ce que l'on pourrait appeler la thèse du façonnement patriarcal de la demande prostitutionnelle n'a pas pour seul enjeu d'ancrer la revendication de sa pénalisation dans la lutte féministe. Elle permet elle aussi de généraliser et d'équilibrer la dénonciation qu'elle porte par grandissement de sa victime. Si l'invocation d'un client « homme ordinaire » permet d'élargir le statut de persécuteur à, potentiellement, l'ensemble des individus de sexe masculin, la référence au patriarcat permet symétriquement d'attribuer le statut de victime à la totalité de la population féminine. D'où l'insistance des féministes et abolitionnistes sur le caractère « collectif » et «systémique » d'une prostitution qui met en jeu la globalité des rapports de genre. Ainsi, pour Attac, « la prostitution contribue à maintenir toutes les femmes dans un statut inférieur » (2008, 61), tandis que pour Alternative libertaire la prostitution est une monétarisation du patriarcat. Elle opprime donc toutes les femmes (Ferrand, 2010, 15). À son tour, cette extension de la problématisation à l'ensemble des femmes présente l'avantage de réfuter le reproche, 
couramment adressé par les organisations de «travailleuses du sexe », d'usurpation de la parole des premières concernées, à savoir les prostituées. Si la prostitution ne concerne pas seulement celles qui l'exercent mais toutes les femmes, ces dernières ont autant de légitimité qu'elles à prendre position sur le sujet. Si elle n'est pas qu'une simple affaire de négociation interindividuelle entre prostituée et client, elle devient un enjeu proprement politique.

\section{III - Une co-construction politique}

Le processus de constitution du problème des clients de prostituées peut gagner à être envisagé à l'aune de ce que Malcolm Spector et John Kitsuse (2009) ont appelé l'histoire naturelle des problèmes sociaux, qui en distingue les étapes successives. La première $\mathrm{a}$, conformément à leur modèle, consisté en une définition publique de la prostitution comme une réalité offensante et déplorable. Lui a succédé son appropriation par les pouvoirs publics, en l'occurrence par le ministre de l'Intérieur qui, en 2002, s'est appuyé sur l'argumentaire développé par les abolitionnistes et les féministes pour donner au problème sa propre «solution »- en l'occurrence sous forme de la loi de sécurité intérieure (LSI) qui pénalise non les clients mais les prostituées coupables de racolage (Mathieu, 2014, 227-241). Ainsi que le relèvent Spector et Kitsuse, cette deuxième étape assiste fréquemment à une dépossession des porteurs originels du problème au profit d'autorités entendant le résoudre par des moyens éloignés des revendications initiales. En découle la troisième étape du processus, sous forme d'une remobilisation d'entrepreneurs de morale insatisfaits de la réponse politique apportée à leurs doléances. Dans le cas présent, elle se traduit par une combativité redoublée en faveur de la pénalisation des clients dont la campagne «Abolition 2012 », animée par plusieurs dizaines d'associations féministes et abolitionnistes, fournit la principale expression.

Pour éclairante qu'elle soit, la perspective de Spector et Kitsuse n'est pas totalement satisfaisante. L'expression malheureuse d'histoire naturelle, tout d'abord, tend à poser comme successives et nécessaires des étapes dans les faits plus imbriquées et aléatoires. Mais c'est surtout la césure postulée entre groupes mobilisés et autorités institutionnelles qui prête dans le cas présent à débat, puisque l'analyse fait apparaître des actrices institutionnelles présentes dans l'espace militant tandis que l'on assiste à la cooptation institutionnelle de personnalités du mouvement. Plus globalement, la problématisation présente ne correspond pas au modèle interpellation militante-réponse politique proposé par les deux auteurs mais davantage à un travail de consolidation et de légitimation mutuelle entre partenaires aux schèmes de perception communs.

\section{1) Consolidations croisées}

Les premières évocations du «modèle suédois » sont, au sein de l'espace abolitionniste et féministe, relativement prudentes. Dans son rapport de 2002, Marcovich avance qu'il serait important de reprendre et d'analyser les effets de la loi suédoise "Paix des Femmes" dont les résultats en matière de prévention et de réinsertion semblent aujourd'hui extrêmement performants (2002, s.p.) mais ne va pas jusqu'à faire figurer la pénalisation des clients parmi ses recommandations. De même Legardinier insiste-t-elle surtout, dans sa notice du Dictionnaire critique du féminisme, sur les aspects pédagogiques de la politique suédoise - qui propose aux clients des services d'écoute téléphonique ou des entretiens avec des psychologues - et sur la nécessité d'actions de prévention et d'éducation sur les causes profondes de la prostitution (2000, 166-167). La pénalisation des clients suscite par ailleurs des débats au sein de certains partis de gauche - ainsi des Verts ou de la LCR dont la brochure sur le sujet expose des positions divergentes.

On trouve une prudence similaire dans la première prise de position institutionnelle sur le sujet, le rapport sur la prostitution présenté en 2000 par délégation du Sénat aux droits des femmes et à l'égalité des chances entre les hommes et les femmes. Présidée par la sénatrice Dinah Dericke, cette délégation procède d'une évolution politique importante pour notre sujet, à savoir ce que Laure Bereni et Anne Revillard (2007) ont appelé l'acclimatation institutionnelle des problématiques féministes. La mise en place, en 1999, de délégations parlementaires aux droits des femmes s'inscrit dans un processus qui a également vu la création de l'Observatoire de la parité et l'instauration du poste de déléguée interministérielle aux droits des femmes. Ces 
mesures contribuent à l'apparition d'un féminisme d'État en mesure de politiser les enjeux portés par le mouvement des femmes, dont la prostitution ${ }^{20}$.

La délégation sénatoriale a appuyé sa réflexion sur plusieurs auditions de personnalités parmi lesquelles dominent les abolitionnistes et féministes : M.-V. Louis, le MAPP, le Mouvement du Nid, la Fondation Scelles, la Fédération abolitionniste internationale et la Coordination française du Lobby européen des femmes ont été invités à exposer leurs positions, tandis que des options alternatives n'ont pu être exprimées que par la seule association de prévention du sida Cabiria. Le rapport reprend logiquement la définition abolitionniste du problème des clients, en pointant la nécessité que le "client" prenne conscience : que la prostitution est un échec, non seulement pour la prostituée, mais aussi pour lui ; qu'il s'agit peut-être pour lui d'un acte anodin, sans signification, mais que, pour la prostituée, il s'agit d'une violence ; qu'en recourant à la prostitution, il a toutes chances d'être complice d'un proxénète, et, le cas échéant, d'un trafiquant d'êtres humains (Dericke, 2000-2001, 58). Mais il se garde de préconiser une politique précise à leur destination (Faut-il le responsabiliser par l'éducation ou la pénalisation ?) (ibid., 95) et se cantonne à un appel à approfondir la réflexion.

Rompant avec une longue tradition d'évitement politique du thème prostitutionnel, le « rapport Dericke » est le premier travail parlementaire spécifiquement consacré à la prostitution. Il ne restera pas longtemps isolé et les rapports sur le sujet vont, les années suivantes, se multiplier à un rythme allant s'accélérant : rapport Lazerges-Vidalies sur l'esclavage moderne de 2001, rapport Bouquet-Geoffroy sur la prostitution de 2011, rapport Jouanno-Godefroy sur la situation sanitaire et sociale des prostituées de 2013, rapport Olivier sur la lutte contre le système prostitutionnel de 2013, auxquels il faut ajouter les rapports d'information produits à l'occasion de la discussion de projets ou propositions de $\operatorname{loi}^{21}$ ainsi que la résolution « réaffirmant la position abolitionniste de la France en matière de prostitution » adoptée par l'Assemblée nationale en décembre 2011.

L'élaboration de ces documents institutionnels procède d'une co-construction à la fois militante et politique tant sont étroits les liens entre mouvements abolitionniste et féministe et porteurs de la question prostitutionnelle au sein des arènes institutionnelles. Le multipositionnement, en premier lieu, caractérise plusieurs protagonistes importants du processus, amenés à se côtoyer dans ces sortes de « forums hybrides » que sont les conseils ou observatoires spécialisés, où politiques et anciens politiques se mêlent aux représentants de la « société civile ». Legardinier n'est ainsi pas seulement journaliste à $P \& S$ mais a été membre de l'Observatoire de la parité entre 2002 et 2005. L'actuel secrétaire général du Mouvement du Nid Grégoire Théry est quant à lui membre du Haut conseil à l'égalité entre les hommes et les femmes qui lui a succédé. Celui-ci est présidé par Danièle Bousquet, ancienne députée socialiste, auteure avec Guy Geoffroy du rapport parlementaire sur la prostitution de 2011, ancienne militante du Planning familial qui se réclame toujours du féminisme. Les engagements partisans de nombre de féministes contribuent quant à eux - via, notamment, le CNDF - à une conversion de la plupart des formations de gauche et d'extrême gauche à la pénalisation des clients. C'est en effet par la gauche du champ politique que celle-ci connaîtra sa légitimation.

Il est également important de contextualiser ce processus de ralliement politique à l'option de la pénalisation des clients. Autre lieu de rencontre entre politiques et militants, le colloque sur les « Politiques urbaines face à la prostitution », tenu à Nantes en 2002 et dont l'organisation a été confiée à Marcovich et Legardinier, est intéressant non pas tant pour sa composition exclusivement féministe et abolitionniste côté associatif, mais parce que, côté politique, ce sont surtout des élus en charge des questions de sécurité qui y interviennent. La prostitution s'est en effet imposée, à partir de la fin des années 1990, sur l'agenda des politiques municipales du fait de l'arrivée de prostituées étrangères, réputées sous la coupe de proxénètes mafieux, très visibles dans l'espace public et responsables de nuisances dont se plaignent les riverains. La définition de la prostitution comme violence sexiste appelant une réponse pénale que promeuvent féministes et abolitionnistes rencontre dès lors un écho certain parmi des élus sensibles aux thématiques d'insécurité et disposés à apporter une solution répressive aux nuisances urbaines ${ }^{22}$. 
On sait que cette réponse répressive a dans un premier temps pris une autre direction, avec l'adoption — au niveau local d'abord puis national par la LSI — d'instruments de pénalisation du racolage, visant donc les seules prostituées. L'option de la sanction des clients a fait sa première véritable apparition politique lors du débat préalable à l'adoption de la LSI, par une proposition de loi du député socialiste Christophe Caresche. Ce texte a permis à un PS depuis peu revenu dans l'opposition de se distinguer de la pénalisation du racolage préconisée par le ministre de l'Intérieur tout en maintenant la question prostitutionnelle dans un cadre pénal. Que C. Caresche soit au même moment adjoint en charge de la sécurité et de la prévention au sein d'une mairie de Paris elle aussi confrontée aux nuisances causées par la présence de prostituées dans ses zones résidentielles suggère que la volonté de les sauver d'une violence sexiste pouvait se combiner à celle de rendre leur activité plus discrète. Envisagée dans ses modalités pratiques, la pénalisation des clients apparaît en effet comme le double inversé de celle du racolage, dont est attendue la même invisibilisation de la prostitution par son expulsion des zones urbaines au profit de lieux d'exercice plus discrets (Mathieu, 2013).

La perspective d'une alternance lors de l'élection présidentielle de 2012 a suscité la remobilisation de l'espace militant abolitionniste et féministe. Le bilan critique des effets de la LSI a favorisé la défense d'une option alternative visant non plus les prostituées mais leurs clients tout en restant dans une inspiration pénale. Mais la promotion de la pénalisation s'est surtout réalisée au travers de la campagne "Abolition 2012 », initiée au sein de l'espace militant tout en s'appuyant sur des ressources institutionnelles. C'est ainsi à l'occasion d'un colloque organisé en février 2010 par le Mouvement du Nid et 17 associations partenaires, constitués en un "Front de refus du système prostitutionnel », que la campagne a été impulsée ${ }^{23}$. Que ce colloque se soit tenu au Palais Bourbon avec la participation active de plusieurs parlementaires - au premier rang lesquels Danièle Bousquet - atteste l'achèvement de l'intrication des logiques militantes et institutionnelles. La mise en place dans sa foulée de la mission d'information sur la prostitution présidée par Bousquet en a constitué le premier résultat concret.

\section{2) Le travail politique}

On sait que la campagne «Abolition 2012 » a connu une étape décisive avec la proposition de loi « renforçant la lutte contre le système prostitutionnel », votée par l'Assemblée nationale le 4 décembre 2013 et qui prévoit une interdiction d'achat d'acte sexuel en créant une contravention sanctionnant le recours à la prostitution. L'objet de cette section est de s'attarder sur le travail proprement politique de validation législative de la problématisation portée par les abolitionnistes et les féministes.

44 On doit tout d'abord remarquer qu'une pénalisation des clients de prostituées existe depuis la loi du 4 mars 2002, qui instaure une peine de trois ans de prison et de $45000 €$ d'amende pour les clients de mineurs prostitués de 15 à 18 ans. Le fait que cette mesure ait pris place au sein d'un dispositif législatif sur l'autorité parentale, et abordant notamment la maltraitance, confirme l'association entre violence et prostitution. La LSI a, l'année suivante, complété le dispositif en prévoyant les mêmes peines pour le recours à une prostituée lorsque celleci présente une particulière vulnérabilité due à une maladie, une infirmité, une déficience physique ou psychique ou un état de grossesse. Cette pénalisation des clients de prostituées mineures ou vulnérables a été préparée par le rapport Lazerges-Vidalies, remis en 2001 et consacré à la lutte contre l'esclavage moderne. Plusieurs pages y étaient dédiées à la prostitution et la commission chargée de son élaboration avait auditionné le Nid et le MAPP. Le rapport arguait alors que s'il est légitime de poursuivre et de condamner les personnes se livrant, hors de nos frontières, aux pratiques de "tourisme sexuel", il est également impérieux d'assurer la répression des agissements comparables qui se déroulent désormais sur notre propre sol et regrettait que notre droit pénal ne réprime le client d'une personne mineure prostituée que si celle-ci est âgée de moins de 15 ans, la "majorité sexuelle" débutant au-delà de cet âge (Lazerges, Vidalies, 2001, 203).

45 Défendue, on l'a vu, par le PS, l'extension de la pénalisation aux clients de prostituées majeures n'est évoquée que sur un mode mineur lors du débat préalable à l'adoption de la LSI. Rédigé 
par l'UMP Marie-Jo Zimmermann, le rapport d'information du Sénat invoque à ce sujet le manque de recul sur les effets de la politique suédoise et la différence de contexte culturel qui sépare les deux pays. Il s'approprie cependant l'argumentaire abolitionniste-féministe en appelant à une réflexion sur la violence que constitue l'achat des services sexuels et au-delà le soutien aux réseaux mafieux qu'il implique (Zimmermann, 2002, 29).

L'appropriation est beaucoup plus nette dans le rapport Bousquet-Geoffroy. Publié en avril 2011, il rend compte d'un important travail de la part d'une commission composée de députés tant PS qu'UMP. Si 200 personnalités aux positions contrastées sur le sujet ont été auditionnées, ce sont les abolitionnistes et les féministes qui sont les plus fréquemment cités. S'agissant plus spécifiquement du client, le rapport avance à son tour qu'il est un « acteur central mais longtemps occulté du système prostitutionnel », que « le constat est simple : sans clients la prostitution n'existerait pas » et, citant Bouamama, que la pornographie peut (...) être vue comme "une étape fréquente du processus de devenir-client" (Bousquet, Geoffroy, 2011, 214 et 239). Considérant que pénaliser les clients c'est leur faire comprendre qu'ils participent à une forme d'exploitation de la vulnérabilité d'autrui (ibid., 232) et s'appuyant sur les constats d'un déplacement à Stockholm, la commission propose de «créer un délit sanctionnant le recours à la prostitution ». Cette préconisation n'est qu' une parmi trente autres, relatives à la protection des victimes de la traite et à l'accompagnement social des prostituées, mais elle figure à la première place et c'est elle qui est la plus commentée.

L'approche des échéances électorales empêche l'adoption des mesures préconisées par le « rapport Bousquet-Geoffroy » mais débouche néanmoins en décembre 2011 sur l'adoption de la résolution « réaffirmant la position abolitionniste de la France ». Celle-ci affirme notamment, en se référant à la politique suédoise et sans craindre les pléonasmes, que « la loi doit clairement marquer la responsabilité de chacun dans la perpétuation du système prostitutionnel. (...) Elle doit (...) responsabiliser les clients en leur indiquant clairement qu'eux aussi ont une part de responsabilité ». Ce texte est adopté à l'unanimité de l'Assemblée nationale, attestant du caractère désormais politiquement consensuel des positions féministesabolitionnistes, auxquelles les formations de droite se sont converties sous l'influence de Geoffroy.

48 Le processus est relancé dès le retour du PS au pouvoir, puisque c'est quelques semaines après son entrée en fonction que la nouvelle ministre des Droits des femmes Najat VallaudBelkacem déclare dans les colonnes du Journal du dimanche que son objectif est de voir la prostitution disparaitre et invoque l'unanimité des partis sur la pénalisation des clients. Malgré un déplacement à Stockholm en novembre 2012, la ministre parait faire marche arrière en février 2013 en affichant dans le magazine Causette une position plus hésitante. L'option de la pénalisation des clients ne fait pas l'unanimité au sein du secteur associatif comme du mouvement féministe et, par prudence, le gouvernement confie aux parlementaires la tâche de défendre cette option. La députée PS Maud Olivier se charge du dossier et remet en septembre 2013, après l'incontournable déplacement à Stockholm, un rapport qui s'inscrit dans la continuité directe de l'appropriation, par le rapport Bousquet-Geoffroy, de la rhétorique féministe-abolitionniste. Ici encore, Le client, c'est celui dont on ne parle jamais. Pourtant, sans lui, "la prostitution et la traite des êtres humains à des fins d'exploitation sexuelle n'existeraient pas". Ici encore, sont invoqués l'influence de la pornographie violente sur la construction des représentations de la sexualité des jeunes, la responsabilité des clients dans la perpétuation des trafics et le fait qu'acheter un acte sexuel constitue une violence faite à autrui ce qui suppose d'accorder une certaine gravité à cet acte et donc à sa sanction (Olivier, 2013, 13, 96, 106 et 113).

La fusion des discours militants et institutionnels est plus évidente encore dans le rapport remis par Brigitte Gonthier-Maurin en juin 2014 à l'occasion de l'examen de la proposition de loi par le Sénat. Une nouvelle fois est assuré que les rares études disponibles sur les consommateurs de prostitution mettent en évidence le fait que le client est de tous les âges et de toutes les conditions sociales : c'est "Monsieur tout le monde" (Gonthier-Maurin, 2014, 27). Une nouvelle fois sont invoqués les liens entre prostitution et pornographie (ibid., 7). Une nouvelle fois est affirmé que les clients des personnes prostituées sont des hommes, qui 
considèrent le corps de ces personnes comme une marchandise (19), que la prostitution se caractérise, du côté de l'acheteur, par le malaise et le mal-être (30) et qu'aucune pulsion sexuelle n'autorise à louer un corps humain (25). Une nouvelle fois est invoquée la vocation « pédagogique » de la sanction des clients qui consisterait à poser une limite simple et de rappeler un principe essentiel : il n'est pas de tolérable qu'un être humain achète les services sexuels d'un autre être humain (48).

Cet argumentaire rodé semble pourtant avoir rencontré une limite lorsque la commission spéciale du Sénat a estimé, dans son rapport de juillet 2014 sur la proposition de loi (Meunier, 2014), qu'il existait un risque réel que l'incrimination des clients place les personnes prostituées dans des conditions plus précaires et dangereuses. L'article prévoyant la pénalisation de l'achat de services sexuels a par conséquent été retiré du texte soumis à l'examen des sénateurs le 30 mars 2015. La droite étant en outre redevenue majoritaire au sein de la Haute assemblée, les débats puis le vote ont confirmé cette éviction de la pénalisation des clients au profit d'un maintien de la sanction du racolage. Ce revirement a suscité l'ire de la mouvance abolitionniste-féministe - le Mouvement Nid parlant de « vote réac, déshonorant et irresponsable $»^{24}$ - qui peut cependant toujours compter sur la navette parlementaire (à savoir le réexamen du texte par l'Assemblée nationale et son vote en des termes identiques par les deux assemblées) pour permettre son adoption finale.

La pénalisation des clients de prostituées n'est, au moment où ces lignes sont écrites, pas encore instaurée en France. Mais, bien qu'encore inachevé, le processus étudié ici aura déjà obtenu un succès par l'adoption définitive, le 4 août 2014, de la Loi pour l'égalité réelle entre les femmes et les hommes. Son article $1^{\text {er }}$ stipule en effet à son deuxième alinéa que la politique pour l'égalité entre les femmes et les hommes comporte notamment des actions visant à renforcer la lutte contre le système prostitutionnel - sans toutefois se prononcer sur les moyens de cette lutte. Cette loi ne fait pas qu' institutionnaliser une formulation et une approche - celle qui fait de la prostitution un «système »-directement issues de l'espace féministe et abolitionniste. Elle entérine également son appréhension comme un point d'achoppement de l'égalité entre hommes et femmes, autrement dit comme une question de genre. Déjà en germe dans le transfert administratif, au début des années 2000, du dossier prostitution au service des Droits des femmes, cette approche ne se substitue toutefois pas à d'autres appréhensions du phénomène prostitutionnel, par ailleurs objet d'une multiplicité de politiques publiques : comme problème social, comme enjeu de santé publique et comme cible d'action policière. Que cette dernière puisse être enrôlée dans une politique visant, au nom du féminisme, à l'abolition de la prostitution peut être rapproché du mouvement plus général de pénalisation des questions sociales (Wacquant, 2004). Alors que le ralliement de la France à l'abolitionnisme a présidé, en 1960, à la mise en place d'un secteur spécialisé du travail social chargé d'assister les prostituées et de les accompagner vers la réinsertion, c'est aujourd'hui à la police qu'il est demandé de faire disparaître la prostitution en sanctionnant ses clients. Cette évolution suggère également que l'institutionnalisation du féminisme s'accompagne, en France comme aux États-Unis (Bernstein, 2010), de son déplacement d'une conception redistributive de la justice vers une vision principalement punitive de celle-ci. En passant du statut d'inadaptée sociale à celui de victime de violence, la prostituée voit le contexte économique et social de son activité s'estomper au profit d'une condamnation avant tout morale. La prostitution n'est plus ici la résultante de structures sociales inégalitaires mais une interaction intrinsèquement violente entre une victime et un coupable, redevable d'une action punitive individualisée.

\section{Bibliographie}

Attac, 2008, Mondialisation de la prostitution, atteinte globale à la dignité humaine, Paris, Mille-et-unenuits.

Bajos N., Pryen S., Warszawski J., Serre A., 1997, Sexualité vénale et gestion du risque de transmission sexuelle du sida, Sciences sociales et santé, 15, 3, 31-60. 
Banaszak L.A., 2010, The Women's Movement Inside and Outside the State, Cambridge, Cambridge University Press.

Becker H., 1985 [1963], Outsiders. Études de sociologie de la déviance, Paris, Métailié.

Bereni L., 2012, Penser la transversalité des mobilisations féministes : l'espace de la cause des femmes, in Bard C. (dir.), Les Féministes de la deuxième vague, Rennes, PUR, 27-41.

Bereni L., Revillard A., 2007, Des quotas à la parité : "féminisme d'État" et représentation politique (1974-2007), Genèses, 67, 5-23.

Berlière J.-M., 1992, La Police des mœurs sous la III ${ }^{\mathrm{e}}$ République, Paris, Seuil.

Bernstein E., 2010, Militarized Humanitarianism Meets Carceral Feminism: The Politics of Sex, Rights, and Freedom in Contemporary Antitrafficking Campaigns, Signs, 36, 1, 45-71.

Boltanski L., 1990, L’Amour et la justice comme compétences, Paris, Métailié.

Bouamama S., Legardinier C., 2006, Les Clients de la prostitution. L'enquête, Paris, Presses de la Renaissance.

Bousquet D., Geoffroy G., 2011, Prostitution : l'exigence de responsabilité. En finir avec le mythe du «plus vieux métier du monde », Assemblée nationale, Commission des lois, $n^{\circ} 3334$.

Chaumont J.-M., 2009, Le Mythe de la traite des blanches. Enquête sur la fabrication d'un fléau, Paris, La Découverte.

Charpenel Y. (dir.), 2012, Rapport mondial sur l'exploitation sexuelle, Paris, Economica.

Christine, 2010, Ce très ordinaire désir de domination, Alternative libertaire, 197, 11.

Coquart E., Huet P., 2000, Livre noir de la prostitution, Paris, Albin Michel.

Corbin A. 1982, Les Filles de noce. Misère sexuelle et prostitution (19 ${ }^{\mathrm{e}}$ siècle), Paris, GarnierFlammarion.

Dericke D., 2000-2001, Les Politiques publiques et la prostitution. Rapport d'information sur l'activité de la délégation aux droits des femmes et à l'égalité des chances entre les hommes et les femmes pour l'année 2000, Sénat, $n^{\circ} 209$.

Ferrand A., 2010, Pour l'abolition. Arrêter le mensonge, Alternative libertaire, 197, 15.

Florin O., 2012, A Particular Kind of Violence: Swedish Social Policy Puzzles of a Multipurpose Criminal Law, Sexual Research and Social Policy, 9, 3, 269-278.

Gemähling P., 1933, La Réglementation administrative de la prostitution jugée d'après les faits, Bordeaux, éditions du Relèvement social et Paris, Recueil Sirey.

Gilbert G., Henry E., 2012, La définition des problèmes publics : entre publicité et discrétion, Revue française de sociologie, 53, 1, 35-59.

Gonthier-Maurin B., 2014, Rapport d'information fait au nom de la Délégation au droit des femmes et à l'égalité des chances entre les hommes et les femmes sur la proposition de loi n ${ }^{\circ} 207$ (2013-2014), adoptée par l'Assemblée nationale, renforçant la lutte contre le système prostitutionnel, Sénat, ${ }^{\circ} 590$.

Gould A., 2001, The Criminalization of Buying Sex: the Politics of Prostitution in Sweden, Journal of Social Policy, 30, 3, 437-456.

Grignon C., 1991, Racisme et racisme de classe, bis, Critiques sociales, 2, 3-12.

Gusfield J., 2009 [1981], La Culture des problèmes publics, Paris, Economica.

Haines H., 2013, Radical flank effect, in Snow D. A., Della Porta D., Klandermans B., McAdam D. (dir.), The Wiley-Blackwell Encyclopedia of Social and Political Movements, Malden and Oxford, Blackwell.

Henry E., 2009, Construction des problèmes publics, in Fillieule O., Mathieu L., Péchu C. (dir.), Dictionnaire des mouvements sociaux, Paris, Presses de Science Po, 146-154.

Hubbard P., Matthews R., Scoular J., 2008, Regulating sex work in the EU: prostitute women and the new spaces of exclusion, Gender, Place and Culture, 15, 2, 137-152.

Kulick D., 2003, Sex in the New Europe. The Criminalization of Clients and Swedish Fear of Penetration, Anthropological Theory, 3, 2, 199-218.

Lazerges C., Vidalies A., 2001, Rapport de la mission d'information commune sur les diverses formes de l'esclavage moderne, Assemblée nationale, $\mathrm{n}^{\circ} 3459$.

LCR, 2006, Prostitution : comment (s')en sortir, Dossier Rouge.

Legardinier C., 1996, La Prostitution, Toulouse, Milan. 
Legardinier C., 2000, Prostitution 1, in Hirata H., Laborie F., Le Doaré H., Sénotier D. (dir.), Dictionnaire critique du féminisme, Paris, PUF, 2004 (2éd.), 161-166.

Legardinier C., 2011, Prostitution : les "clients" tremblent pour leurs petits privilèges, Prostitution et société [http://www.prostitutionetsociete.fr/eclairage/point-de-vue/prostitution-les-clients-tremblent] (consulté le 19 août 2014).

Legardinier C., 2012, "Clients" : un droit de l'homme en question, Chronique féministe, n 109, 49-53.

Louis M.-V., 1999 [1997], Le corps humain mis sur le marché, Le Monde diplomatique, Manière de voir (Le Monde diplomatique), 44, 13-15.

Louis M.-V., 2004, Prostitution, in Lopez G., Tzitzis S. (dir.), Dictionnaire de sciences criminelles, Paris, Dalloz, 759-761.

Marcovich M., 2002, Le système de la prostitution : une violence à l'encontre des femmes, Commission nationale contre les violences envers les femmes.

Marcovich M., 2006, La traite des femmes dans le monde, in Ockrent C. (dir.), Le Livre noir de la condition des femmes, Paris, XO, 449-490.

Mathieu L., 2001, Mobilisations de prostituées, Paris, Belin.

Mathieu L., 2002, Rapport au politique, dimensions cognitives et perspectives pragmatiques dans l'analyse des mouvements sociaux, Revue française de science politique, 52, 1, 75-100.

Mathieu L., 2013, Genèse et logiques des politiques de prostitution en France, Actes de la recherche en sciences sociales, 198, 4-21.

Mathieu L., 2014, La Fin du tapin. Sociologie de la croisade pour l'abolition de la prostitution, Lormont, François Bourin.

Mathieu L., à paraître, Le mouvement abolitionniste français dans l'après-guerre. Recompositions et reconversions de la croisade contre la prostitution réglementée, Déviance et Société.

Meunier M., 2014, Rapport fait au nom de la commission spéciale sur la proposition de loi, adoptée par l'Assemblée nationale, renforçant la lutte contre le système prostitutionnel, Sénat, nº 697.

Montreynaud F., 1993, Amours à vendre. Les dessous de la prostitution, Grenoble, Glénat.

Montreynaud F., 1999, La prostitution, un droit de l'homme ?, Manière de voir, n 44, 19-21.

Montreynaud F., 2002, Table ronde. Prostitution et violence, in Collectif, De nouveaux défis pour le féminisme, actes du Forum du Collectif national pour les droits des femmes, Pantin, Le Temps des cerises, 195-198.

Mossuz-Lavau J., 2015, La Prostitution, Paris, Dalloz.

Olivier M., 2013, Rapport fait au nom de la Délégation aux droits des femmes et à l'égalité des chances entre les hommes et les femmes sur le renforcement de la lutte contre le système prostitutionnel, Assemblée nationale, $\mathrm{n}^{\circ} 1360$.

Regard F. (dir.), 2013, Féminisme et prostitution dans l'Angleterre du XIX ${ }^{\mathrm{e}}$ siècle : la croisade de Josephine Butler, Lyon, ENS éditions.

Rozier J., 2002, Rapport d'information fait au nom de la Délégation au droit des femmes et à l'égalité des chances entre les hommes et les femmes sur le projet de loi n 30 (2002-2003) pour la sécurité intérieure, Sénat, $\mathrm{n}^{\circ} 34$.

Safar D ${ }^{\mathrm{r}}$, 1973, Psychologie des acteurs, Chronique sociale de France, 6, 22-26.

Spector M., Kitsuse J., 2009 [1977], Constructing Social Problems, New Brunswick, N.J., Transaction Publishers.

Union française pour le relèvement de la moralité publique, 1936a, Une Institution qui déshonore notre pays : la réglementation officielle de la prostitution, Union française pour le relèvement de la moralité publique, Paris.

Union française pour le relèvement de la moralité publique, 1936b, Bulletin annuel de l’Union temporaire contre la prostitution réglementée et la traite des êtres humains, $\mathrm{n}^{\circ} 6$.

Union française pour le relèvement de la moralité publique, 1950, Rénovation. Organe trimestriel du Cartel d'action morale et sociale, $\mathrm{n}^{\circ} 11$.

Wacquant L., 2004, Punir les pauvres. Le nouveau gouvernement de l'insécurité sociale, Marseille, Agone. 
Zimmermann M.-J., 2002, Rapport d'information fait au nom de la Délégation au droit des femmes et à l'égalité des chances entre les hommes et les femmes sur le projet de loi adopté par le Sénat après déclaration d'urgence ( $\left.n^{\circ} 381\right)$ pour la sécurité intérieure, Assemblée nationale, $n^{\circ} 459$.

\section{Notes}

1 On se focalisera ici sur le seul niveau français pour laisser de côté, faute de place, le niveau européen où se jouent également des processus institutionnels influents.

2 Les deux notions relèvent de démarches similaires, attentives aux processus de construction sociale qui les font accéder à une forme d'existence ; Gusfield les distingue en pointant que tous les problèmes sociaux ne deviennent pas des objets de conflit ou de controverse dans les arènes de l'action publique, ce qui serait le propre des problèmes publics (Gusfield, 2009, 5). Pour une discussion des rapports entre les deux concepts, voir Henry (2009).

3 On emploiera ici le terme de prostituée uniquement au féminin, conformément à l'appréhension essentiellement féminine de cette activité qui caractérise la rhétorique féministe-abolitionniste. Si celleci reconnaît des formes masculines de prostitution, elle les intègre à sa bi-catégorisation genrée (femmesprostituées $v s$ hommes-clients) en arguant d'une «féminisation» des hommes qui se livrent à la prostitution.

4 Notons que l'abolitionnisme dont il sera question ici se pose en héritier direct du mouvement pour l'abolition de l'esclavage, auquel certains de ses fondateurs ont participé, et émane originellement des mêmes milieux sociaux, i.e. de la bourgeoisie protestante britannique. En découle, sur le plan des discours, la fréquente assimilation de la prostitution à un esclavage, particulièrement sensible au moment de la croisade contre la «traite des blanche » posée en équivalence aux traites négrières (Chaumont, 2009). En revanche, on ne trouve pas chez lui de référence explicite aux courants favorables à l'abolition des sanctions pénales ou de la prison, et l'emploi du même terme d'abolitionnisme paraît référer à des enjeux distincts.

$5 \mathrm{Du}$ côté de cet autre acteur majeur de l'abolitionnisme français de l'immédiat aprèsSeconde Guerre mondiale qu'est le Cartel d'action morale et sociale (héritier de la Ligue française pour le relèvement de la moralité publique), c'est le contexte culturel général qui est davantage incriminé dans le façonnement de la demande prostitutionnelle. Le Cartel n'a ainsi de cesse de vilipender le climat d'érotisme et de violence complaisamment entretenu par la littérature et le cinéma. Ainsi demande-t-il aux pouvoirs publics et au parquet « de combattre le cinéma corrupteur, la littérature et l'affichage pornographiques, la multiplication exagérée des dancings et des bars louches, des boîtes de nuit et des concours de beauté. (...) Nous demandons enfin à tous, étudiants et jeunes ouvriers, soldats ou chefs de famille, de ne plus considérer la femme comme une bête de somme ou un instrument de plaisir » (Rénovation, $1950, \mathrm{n}^{\circ} 11$, sans pagination).

6 À noter cependant la brochure «Au marché du sexe, client qui es-tu ? » qui en 1993 regroupe les actes de la $3^{\text {e }}$ université d'automne du mouvement le Cri. Mais cette réflexion émanant d'une association dominée au sein de l'espace abolitionniste reste alors isolée.

7 Le Collectif national droit des femmes, notamment, est constitué en janvier 1996, dans la foulée de la manifestation de novembre 1995 pour la défense du droit à l'avortement et à la contraception.

8 Ce système désigne pour la sociologue un système de domination marchande sur les sexes, sur les corps et donc sur les êtres humains qui, par la force, la contrainte et la violence légitimées par le droit, crée les conditions d'une mise en relation de "clients" auxquels des proxénètes - personnes physiques et morales - garantissent, contre rémunération, la possibilité d'un accès marchand aux sexes d'autres personnes, de sexe féminin dans la grande majorité des cas, femmes adultes, adolescentes, petites filles (Louis, 2004).

9 Rapport de 2003 du ministère suédois de l'Industrie, de l'Emploi et de la Communication, cité dans Hubbard, Matthews, Scoular (2008, 143). Sur la politique suédoise, voir également Gould (2001); Kulick (2003) et Florin (2012).

10 Notamment à l'occasion de la publication, sous son égide, du Livre noir de la prostitution (Coquart, Huet, 2000) et de l'organisation la même année d'un colloque à l'UNESCO intitulé « Peuple de l'abîme : la prostitution aujourd'hui».

11 Les Équipes d'action sont habilitées à se porter partie civile dans les procès de souteneurs et ont développé une expertise sur les différents aspects juridiques de la prostitution. La Fondation Scelles est aujourd'hui présidée par un magistrat. 
12 On n'a ainsi trouvé aucune citation directe de l'article de référence sur le sujet de Bajos et al. (1997). Sont par contre amplement évoqués (mais rarement explicitement cités, faute sans doute de traduction) les travaux menés en Suède dans les années 1980 par Sven-Axel Mansson.

13 Voir [http://www.solidaires.org/article31535.html] (consulté le 30 août 2014).

14 « Les motivations des prostitueurs », Prostitution et société, 2009 ; [http:// www.prostitutionetsociete.fr/eclairage/acteurs/les-motivations-des-prostitueurs] (consulté le 21 août 2014).

15 Le pendant de la violence exercée par les clients est le statut de victime accordé aux prostituées et l'insistance, dans le portrait qui en est livré, sur les stress post-traumatiques et autres phénomènes de « décorporalisation » les affectant.

16 «France : la violence d'un État proxénète », sur [http://www.osezlefeminisme.fr/article/france-laviolence-d-un-etat-proxenete] (consulté le 21 août 2014).

17 On trouve le texte de la pétition, diffusée en France par le MAPP, sur [http://sisyphe.org/spip.php? article2226] (consulté le 30 août 2014).

18 Attac met ainsi en cause «l'influence du groupe masculin, les liens avec l'alcool et les sorties du samedi soir, la peur et l'ignorance de l'autre sexe, l'image des femmes et de leur sexualité » dans le recours à la prostitution (Attac, 2008, 55).

19 La stigmatisation féministe-abolitionniste des clients présente ainsi nombre de similarités avec celle du racisme populaire dont Claude Grignon a relevé les traits et la logique : Haine, rage, agressivité, violence : les mots que les spécialistes de l'antiracisme utilisent pour stigmatiser le "racisme populaire" (...) ne sont autres que les mots du racisme de classe ordinaire. (...) Les sentiments attribués aux "basses" classes sont automatiquement dévalorisés comme bas (vils, ignobles, primitifs, etc.) et les sentiments socialement dévalorisés comme bas sont automatiquement et exclusivement attribués aux “basses" classes (Grignon, 1991, 8).

$20 \mathrm{La}$ tendance à la féminisation du personnel politique, loi sur la parité aidant, a contribué à légitimer l'appropriation institutionnelle de la question prostitutionnelle, historiquement délaissée car jugée politiquement indigne (Corbin, 1982) et surtout évoquée, sur la période récente, au travers de positions de parlementaires (majoritairement hommes) tentant un coup d'éclat médiatique en se déclarant favorables à la réouverture des maisons closes (Mathieu, 2014, 217-218).

21 Voir notamment Zimmermann (2002); Rozier (2002); Gonthier-Maurin (2014) et Meunier (2014).

22 Les actes de ce colloque sont accessibles sur le site [http://www.grandesvilles.org/sites/default/ files/publications/actes-colloques/les_politiques_urbaines_face_la_prostitution_13283.pdf] (consulté le 30 août 2014). L'institution policière a cependant rappelé, par la voix de plusieurs syndicats, qu'elle était défavorable à la pénalisation des clients (voir leurs prises de position dans Mossuz-Lavau, 2015, 265-266). Outre les aspects pratiques d'un délit difficile à établir, cette position est peut-être héritée de la réticence historique, rapportée par Berlière (1992), de la police à inquiéter des clients parmi lesquels peuvent se trouver des personnalités à protéger du scandale. Les affaires Ambiel (conseiller du Premier ministre Raffarin poursuivi pour avoir sollicité les services d'une prostituée mineure) et Strauss-Kahn (inquiété pour avoir hébergé des parties fines avec des prostituées) pourraient sur ce point marquer une inflexion de cette attitude, solidaire d'une moindre tolérance d'un monde politique davantage féminisé à l'égard de telles conduites.

23 Les actes du colloque ont été publiés dans le $n^{\circ} 167,2009$, de $P \& S$. Les associations partenaires sont l'Association française des femmes diplômées des universités, la CATW, la Coordination française du Lobby européen des femmes, la Concertation des luttes contre l'exploitation sexuelle, le Centre national d'information sur les Droits des femmes et des familles, le Collectif féministe «Ruptures », le Comité permanent de liaison des associations abolitionnistes, les Élu(e)s contre les violences faites aux femmes, Femmes solidaires, la Fédération nationale solidarité femmes, la Fondation Scelles, la Marche mondiale des femmes, le Mouvement jeunes femmes, le CRI, Rajfire, Regards de femmes et SOS sexisme.

24 [http://www.mouvementdunid.org/PPL-prostitution-au-Senat-un-vote] (consulté le 9 avril 2015).

\section{Pour citer cet article}

\section{Référence électronique}

Lilian Mathieu, « Des monstres ordinaires », Champ pénal/Penal field [En ligne], Vol. XII I 2015, mis en ligne le 10 août 2015, consulté le 18 août 2015. URL : http://champpenal.revues.org/9093 ; DOI : 10.4000/champpenal.9093 


\section{À propos de l'auteur}

Lilian Mathieu

Centre Max Weber, CNRS-ENS de Lyon. Contact : lilian.mathieu@ens-lyon.fr

\section{Droits d'auteur}

(C) Champ pénal

\section{Résumés}

L'article retrace le processus de construction du client en potentiel objet de politique publique, en l'occurrence de politique pénale. Ce processus résulte d'une problématisation portée par une coalition entre les anciennes associations abolitionnistes et des fractions du féminisme centrées sur les enjeux de violence sexiste. Il s'est également appuyé sur un discours expert dépeignant le recours à la prostitution à la fois comme un élément ordinaire de la sexualité masculine et comme l'expression exacerbée d'une brutale libido dominandi. Il a enfin bénéficié de la légitimation internationale apportée par la promotion de la politique suédoise de pénalisation des clients et de l'audience favorable d'un nouveau féminisme d'État.

The paper recounts the process of the construction of the prostitutes' clients as a target of public policy, for instance of a penal policy. This process results from a problematisation that was elaborated by a coalition between old abolitionist organisations and parts of the feminist movement that focus on the issue of sexist violence. It was also based on expert discourse that frames the purchase of sexual services both as an ordinary component of male sexuality and as an exacerbated and brutal libido dominandi. It finally benefited from the international legitimisation and promotion of the Swedish policy that criminalises prostitutes' clients and from the positive appraisal of a new state feminism.

\section{Entrées d'index}

Mots-clés : abolitionnisme, féminisme, prostitution, client, problème public Keywords : abolitionism, feminism, prostitution, clients, public policy 